\title{
Astrogliosis
}

\author{
Michael V. Sofroniew \\ Department of Neurobiology, David Geffen School of Medicine, University of California, \\ Los Angeles, California 90095 \\ Correspondence: sofroniew@mednet.ucla.edu
}

In addition to their many functions in the healthy central nervous system (CNS), astrocytes respond to CNS damage and disease through a process called astrogliosis. For many decades, astrogliosis was sparsely studied and enigmatic. This article examines recent evidence supporting a definition of astrogliosis as a spectrum of heterogeneous potential changes in astrocytes that occur in a context-specific manner as determined by diverse signaling events that vary with the nature and severity of different CNS insults. Astrogliosis is associated with essential beneficial functions, but under specific circumstances can lead to harmful effects. Potential dysfunctions of astrocytes and astrogliosis are being identified that can contribute to, or be primary causes of, CNS disorders, leading to the notion of astrocytopathies. A conceptual framework is presented that allows consideration of normally occurring and dysfunctional astrogliosis and their different roles in CNS disorders.

\begin{abstract}
$A$ strocytes exert many essential functions in Athe healthy central nervous system (CNS) as reviewed and discussed in other articles in this volume. In addition, astrocytes respond to all forms of CNS damage and disease with a variety of potential changes in gene expression, cellular structure, and function. Such responses are commonly referred to as astrogliosis. Although astrogliosis has a long history of descriptive analysis beginning in the late 19th century, there was only sparse interest in, or investigation of, its mechanisms or functions for much of the 20th century. This situation changed gradually over the last two decades when, in parallel with the explosion of information regarding essential astrocyte roles in the healthy CNS, interest has grown in understanding astrogliosis (Pekny and Nilsson 2005; Sofroniew 2009; Sofroniew and Vinters 2010; Kang and Hebert 2011). As summarized in this article, considerable infor-
\end{abstract}

mation has now accrued regarding the mechanisms, functions, and impact of astrogliosis. It is now clear that astrogliosis plays fundamental roles in determining tissue repair and outcome after injury or disease, and has the potential to influence neural function.

\section{WHAT IS ASTROGLIOSIS?}

The term astrogliosis dates back to late 19th and early 20th century neuroanatomists who recognized that astroglia underwent pronounced structural changes in response to CNS damage and disease. At different times, astrogliosis has been assigned various definitions but it has always referred to astrocyte responses to CNS insults. Based on a large cross section of studies in experimental animals and human pathological specimens, we have recently proposed a more detailed working definition of astrogliosis that

Editors: Ben A. Barres, Marc R. Freeman, and Beth Stevens

Additional Perspectives on Glia available at www.cshperspectives.org

Copyright (C) 2015 Cold Spring Harbor Laboratory Press; all rights reserved; doi: 10.1101/cshperspect.a020420

Cite this article as Cold Spring Harb Perspect Biol 2015;7:a020420 
M.V. Sofroniew

encompasses four key features: (1) astrogliosis is a spectrum of potential molecular, cellular, and functional changes in astrocytes that occur in response to all forms and severities of CNS injury and disease; (2) changes undergone by reactive astrocytes vary with severity of the insult along a graded continuum; (3) changes associated with astrogliosis are regulated in a context-specific manner by many different inter- and intracellular signaling molecules; and (4) changes undergone during astrogliosis have the potential to alter astrocyte activities both through gain and loss of functions (Sofroniew 2009; Sofroniew and Vinters 2010). Specific aspects of these features are discussed below.

Various other terms are sometimes used to refer to astrocyte responses to CNS damage or disease, and use of certain terms can vary among investigators. We will use "astrogliosis" and "reactive astrocytes" as general all-inclusive descriptors of all forms of astrocyte responses associated with any form of CNS damage or disease. As discussed in more detail below, these terms encompass astrocyte responses of considerable diversity and heterogeneity. We will not use "activation" or "activated astrocytes" as terms that refer exclusively to astrocyte responses to injury or disease. Astrocytes in healthy tissue continually show physiological activation in the form of transient, ligand-evoked elevations in intracellular calcium $\left(\left[\mathrm{Ca}^{2+}\right]_{i}\right)$ that represent a type of astrocyte excitability, involved in mediating many critical dynamic astrocyte functions, including interactions with synapses and regulation of blood flow as discussed in other articles in this volume. Thus, astrocyte activation can range from physiological contexts in healthy CNS to pathophysiologic contexts that involve responding to CNS injury or disease. In keeping with these definitions, there seems to be no place for the notion of an "activated astrocyte" as a single uniform entity.

\section{MULTICELLULAR RESPONSES TO CNS INSULTS}

Although the focus of this article is on astrogliosis, it is important to emphasize that astro- gliosis does not occur in isolation, but is part of a coordinated multicellular response to CNS insults that includes multiple types of glia as well as neurons and different types of nonneural cells that are intrinsic to the CNS or that enter from the bloodstream (Burda and Sofroniew 2014). Different types of glia that respond to CNS insults include microglia, astrocytes, and NG2-positive oligodendrocyte progenitors (NG2-OPC). Nonneural cells intrinsic to the CNS that respond to insults include endothelia, perivascular fibroblasts, pericytes, and meningeal cells. Cells that enter from the bloodstream after CNS insults include leukocytes, platelets, and other bone marrow-derived cells. Together, these cells have the capacity to produce a vast array of intercellular signaling molecules, and these molecules have the potential to influence the activities and functions of different cell types, including astrocytes. Thus, the response to CNS insults is a complex mixture of events involving the interactions of multiple cell types that change over time (Burda and Sofroniew 2014). Astrocytes take part in these interactions both by receiving instructive signals from other cells and by sending instructive signals that influence other cells (Fig. 1).

\section{ASTROGLIOSIS AS A CONTINUUM OF POTENTIAL RESPONSES}

Astrogliosis has often been referred to as if it were a single uniform entity that is essentially the same in all situations. This is emphatically not the case. A multitude of studies over the past $20 \mathrm{yr}$ have provided compelling evidence that astrogliosis is not a simple stereotypic response, but instead is a finely tuned spectrum of potential changes that range from reversible alterations in gene expression and cellular hypertrophy to pronounced cell proliferation with compact scar formation and permanent tissue rearrangement. Available evidence points toward a wide range of specific molecular signaling mechanisms that regulate specific aspects of astrogliosis, rather than a single stereotypic program that is regulated in an on-off manner (Pekny and Nilsson 2005; Sofroniew 2009; Sofroniew and Vinters 2010; Kang and Hebert 
Astrogliosis

A Astrocytes receive instructions

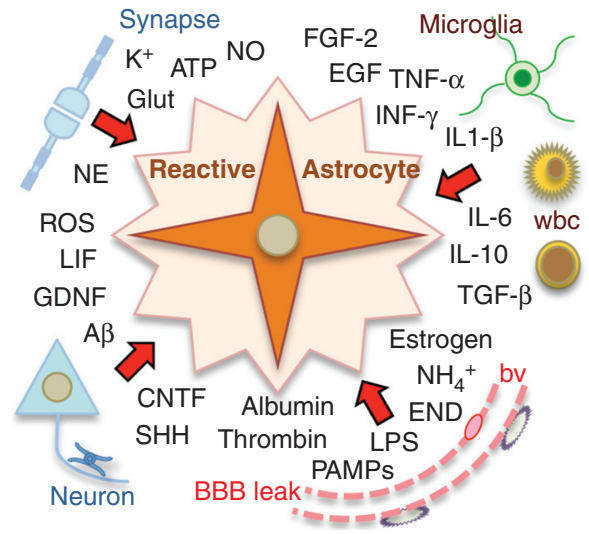

B Astrocytes send instructions

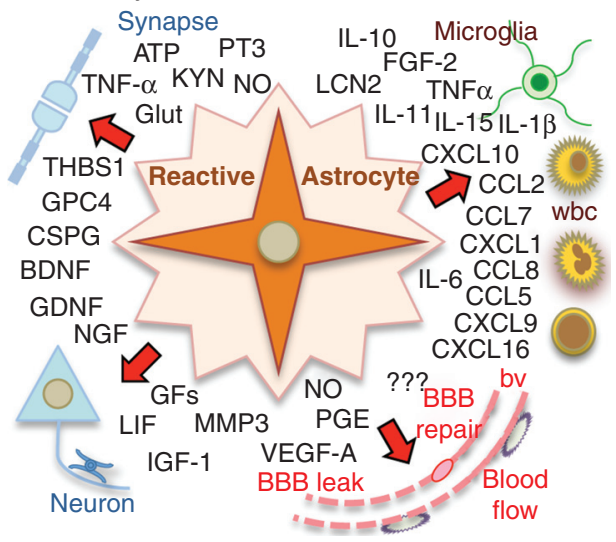

Figure 1. Reactive astrocytes receive and send diverse molecular instructions. Schematic drawings depict examples of diverse molecular signals that instruct reactive astrocytes $(A)$, or that reactive astrocytes release to instruct other cells $(B)$. For abbreviations, see Table 1.

2011). Although there are common features across different forms and intensities of astrogliosis, such as up-regulation of glial fibrillary acidic protein (GFAP) and cellular hypertrophy, even these do not occur in all or none fashions, but are finely regulated along a gradient of intensity that can vary from very small to very intense changes (Fig. 2). Thus, astrogliosis is complex and multivariate, and the changes in astrocytes that are associated with astrogliosis are induced in a context-dependent manner as regulated by many different potential signaling events as summarized in the next sections.

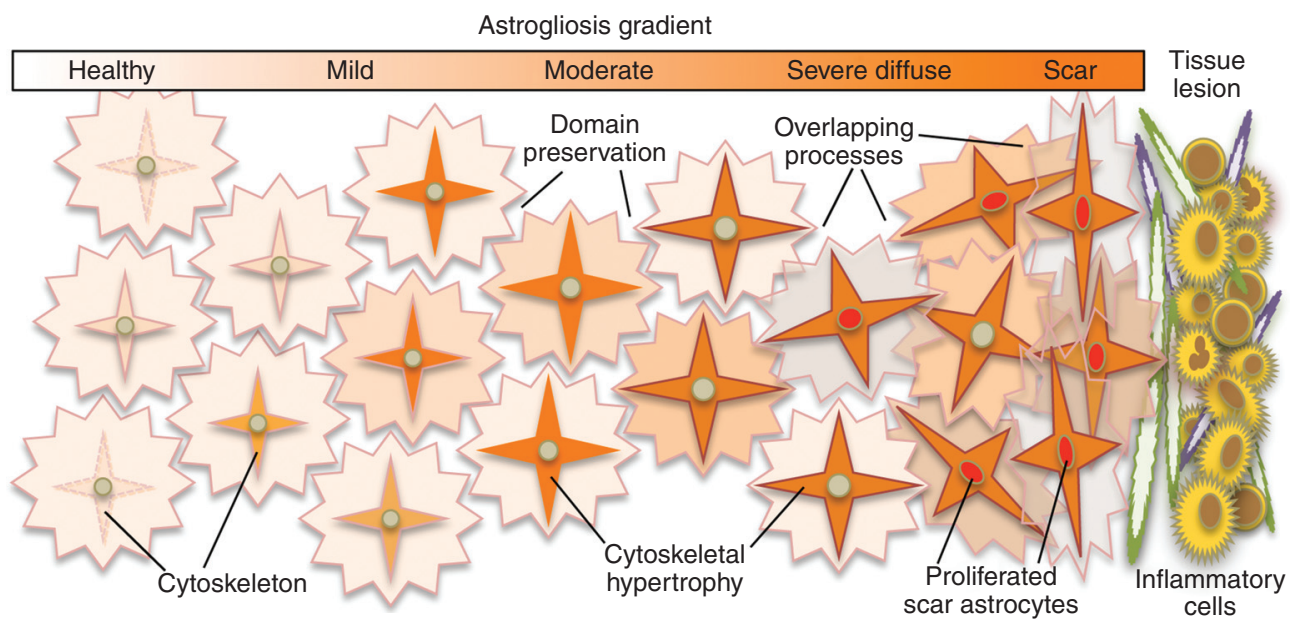

Figure 2. Schema of astrogliosis gradient from mild to moderate to scar. In healthy CNS tissue, many astrocytes do not express detectable levels of the cytoskeletal protein, GFAP. In mild to moderate astrogliosis, most astrocytes up-regulate GFAP and hypertrophy their cytoskeleton but preserve individual domains. In severe diffuse astrogliosis, there is also proliferation (depicted by red nuclei). Compact astroglial scars are comprised of newly proliferated astrocytes with densely overlapping processes that form borders to damaged tissue and inflammation. 
M.V. Sofroniew

\section{SIMPLE CONVENIENT CATEGORIES OF ASTROGLIOSIS}

Changes in astrocyte appearance, molecular expression, and function that are associated with astrogliosis occur along a seamless graded continuum of intensity. Nevertheless, for practical purposes of description, comparison, and discussion, it is convenient to distinguish among several simple categories of astrogliosis as delineated on the basis of changes in structure, molecular expression, and proliferation. These categories include mild to moderate astrogliosis, severe diffuse astrogliosis, and severe astrogliosis with compact scar formation (Fig. 2).

\section{Mild to Moderate Astrogliosis}

Mild to moderate astrogliosis consists of changes (up or down) in astrocyte gene expression together with variable degrees of hypertrophy of cell body and stem processes, without substantive loss of individual astrocyte domains and without astrocyte proliferation (Sofroniew 2009; Sofroniew and Vinters 2010). There is increased expression of the astrocyte intermediate filament protein, GFAP, which is somewhat proportional to the degree of reactivity. The essentially nonoverlapping astrocyte domains found in healthy gray matter are more or less preserved in mild to moderate reactive astrogliosis in brain and spinal cord (Fig. 2) (Wilhelmsson et al. 2006; Wanner et al. 2013). Mild to moderate astrogliosis is generally associated with mild nonpenetrating and noncontusive trauma, or with diffuse innate immune activation (viral infections, system bacterial infections), or occurs in areas that are some distance to focal CNS lesions. After insults that are acute single events, such as traumatic or ischemic injuries, mild to moderate astrogliosis has considerable potential to resolve.

\section{Severe Diffuse Astrogliosis}

Severe diffuse reactive astrogliosis consists of changes (up or down) in gene expression with pronounced up-regulation of GFAP, cellular hypertrophy, dispersed astrocyte proliferation, and some loss of individual astrocyte domains with overlapping of neighboring astrocyte processes (Fig. 2) (Sofroniew 2009; Sofroniew and Vinters 2010). These changes can extend diffusely over substantive areas, and can occur in various situations, such as chronic neurodegenerative insults, diffuse trauma, diffuse ischemia, or certain types of infection. Because there can be considerable tissue reorganization, the potential for resolution and return to normal structure is reduced and there is a high tendency toward long-lasting tissue reorganization (Sofroniew and Vinters 2010).

\section{Severe Astrogliosis with Compact Scar Formation}

Compact astroglial scars derive almost entirely from newly proliferated astrocytes with elongated shapes (Wanner et al. 2013), whose cell processes overlap and intertwine extensively to form compact borders that surround and demarcate areas of tissue damage, necrosis, and inflammation after trauma, stroke, infection, autoimmune-triggered inflammatory infiltration, or neurodegenerative disease (Bush et al. 1999; Faulkner et al. 2004; Drogemuller et al. 2008; Voskuhl et al. 2009; White et al. 2010; Wanner et al. 2013). There may be several possible sources of newly divided scar-forming astrocytes including (1) mature astrocytes that reenter the cell cycle and proliferate (Bush et al. 1999; Buffo et al. 2008; Gadea et al. 2008; Bardehle et al. 2013), (2) NG2 progenitor cells in the local parenchyma (Magnus et al. 2008), or (3) ependymal cell progenitors (Meletis et al. 2008; Barnabe-Heider et al. 2010). Astrocyte scar borders directly interface with and surround a variety of nonneural cell types in the central core of tissue lesions, including perivascular-derived fibroblasts, fibrocytes, pericytes, and other glial cells (Fig. 2) (Bundesen et al. 2003; Herrmann et al. 2008; Sofroniew 2009; Sofroniew and Vinters 2010; Aldrich and Kielian 2011; Reilkoff et al. 2011; Burda and Sofroniew 2014). It is noteworthy that astroglial scar formation is associated with substantive tissue reorganization and structural changes that are essentially permanent and persist even when triggering insults may have resolved. 


\section{HETEROGENEITY OF ASTROCYTES AND REACTIVE ASTROCYTES}

There is growing evidence for and interest in the potential for heterogeneity among astrocytes in healthy CNS not only across different CNS regions, but also locally within the same regions (Zhang and Barres 2010; Tsai et al. 2012). Given that astrogliosis is not a single stereotypic response but is regulated by multiple specific signaling events, there is also growing awareness of heterogeneity among reactive astrocytes at multiple levels (Anderson et al. 2014). For example, adjacent to focal traumatic or ischemic lesions, there is topographic heterogeneity of astrogliosis as regards astrocyte proliferation, morphology, and gene expression with respect to distance from the insult (Sofroniew and Vinters 2010; Wanner et al. 2013). In addition, analysis at the single cell in vivo shows that intermingled reactive astrocytes can show different expression levels of (1) chemokines or cytokines (Hamby et al. 2012), (2) signaling molecules, such as pSTAT3 (Herrmann et al. 2008), or (3) transcription factors that regulate Sonic Hedgehog signaling (SHH) (Garcia et al. 2010). It is also noteworthy that different stimuli of astrocyte reactivity can lead to similar degrees of GFAP up-regulation, whereas causing substantially different changes in transcriptome profiles and cell function (Hamby et al. 2012; Zamanian et al. 2012). Thus, it is not possible to equate simple and uniform measures, such as cell hypertrophy and up-regulation of GFAP expression with a single, uniform concept of astrocyte reactivity. Indeed, certain inflammatory signals can substantively alter reactive astrocyte transcriptome profiles without inducing further changes in GFAP (Hamby et al. 2012). These findings underscore the considerable potential for phenotypic and functional heterogeneity among reactive astrocytes as regulated by specific signaling events.

\section{MULTIPLE MOLECULAR REGULATORS OF ASTROGLIOSIS}

Astrogliosis is not an all-or-none response or stereotypic program regulated by a few simple on-off molecular switches. Instead, astrogliosis is a spectrum of potential changes that occur in a context-specific manner as determined by a myriad of specific potential signaling events that vary with the nature and severity of specific CNS insults. Current evidence indicates that specific aspects of reactive astrogliosis can be regulated separately and individually by a wide variety of intercellular and intracellular signaling and regulatory molecules, as called for in different situations. Combinatorial exposure to multiple signals can markedly alter astrocyte transcriptome profiles and give rise to synergistic effects not predicted by exposure to individual stimuli (Hamby et al. 2012).

\section{Extracellular Signals}

Astrogliosis can be induced, regulated, or modulated by a wide variety of extracellular molecules ranging from small molecules, such as purines, transmitters, and steroid hormones, to large polypeptide growth factors, cytokines, serum proteins, or neurodegeneration associated molecules like $\beta$-amyloid (Table 1 ). These instructive signals can derive from many different sources (Fig. 1A) and can be released by cell damage or cell death, or via specific signaling mechanisms. Many cell types can release molecular regulators of astrogliosis, including (1) local neural and nonneural cells intrinsic to CNS tissue, such as neurons, microglia, oligodendrocyte lineage cells, endothelia, pericytes, fibromeningeal cells, and other astrocytes, as well as (2) nonneural cells that gain entry into the CNS, such as bone marrow-derived leukocytes, fibrocytes, and microbial infectious agents (Fig. 1A) (Burda and Sofroniew 2014). Last but not least, astrogliosis can be regulated by molecules, such as serum proteins, cytokines, or steroid hormones that are produced by cells outside of the CNS, including microbial endotoxins, such as lipopolysaccharides (LPS) (Burda and Sofroniew 2014).

\section{Intracellular Signal Transducers and Regulators}

Many extracellular molecular regulators of astrogliosis have receptor targets that initiate in- 
M.V. Sofroniew

Table 1. Selected examples of molecular regulators of astrogliosis

\begin{tabular}{|c|c|c|}
\hline Categories & Source & Molecules \\
\hline \multicolumn{3}{|c|}{ Extracellular molecular signals } \\
\hline DAMPs & Cell damage & $\begin{array}{l}\text { Adenosine triphosphate (ATP), reactive oxygen } \\
\text { species (ROS), nitric oxide (NO), high-mobility } \\
\text { group box } 1 \text { (HGMB1), etc. }\end{array}$ \\
\hline $\begin{array}{l}\text { Hypoxia and } \\
\text { metabolic stress }\end{array}$ & Ischemia & Oxygen deprivation, glucose deprivation \\
\hline Transmitters & Local neurons & Glutamate (Glut), noradrenalin (NE) \\
\hline \multirow[t]{2}{*}{ Hormones } & Endocrine glands & Estrogens, glucocorticoids \\
\hline & Neurodegeneration & $\beta$-amyloid $(\mathrm{A} \beta)$ \\
\hline \multirow[t]{2}{*}{ PAMPs } & Microbes & LPS, zymosan, dsRNA (viral) \\
\hline & $\begin{array}{l}\text { Systemic metabolic toxicity } \\
\quad \text { (liver failure) }\end{array}$ & Ammonium $\left(\mathrm{NH}_{4}^{+}\right)$ \\
\hline Serum proteins & Bloodstream & Thrombin, albumin, fibrin, etc. \\
\hline $\begin{array}{l}\text { Cytokines and } \\
\text { growth factors }\end{array}$ & $\begin{array}{l}\text { Other glia, local nonneural } \\
\text { cells, infiltrating leukocytes }\end{array}$ & $\begin{array}{l}\text { IL-1 } \beta \text {, tumor necrosis factor (TNF)- } \alpha \text {, interferon } \\
\text { (INF)- } \gamma \text {, IL-6, ciliary neurotrophic factor (CNTF), } \\
\text { leukemia inhibitory factor (LIF), transforming } \\
\text { growth factor (TGF)- } \beta \text {, IL-10, epidermal } \\
\text { growth factor (EGF), fibroblast growth factor } \\
\text { (FGF)-2, Sonic Hedgehog (SHH), endothelin } \\
\text { (END) }\end{array}$ \\
\hline \multicolumn{3}{|c|}{ Astrocyte intrinsic signaling pathways and regulatory mechanisms } \\
\hline Signal transducers & & $\begin{array}{l}\text { STAT3, STAT2, STAT2, JAK2, NF-кB, IRAK, SOC3, } \\
\text { MAP-kinase, SOX9, Nrf2, Olig2, cAMP, Nurr1, } \\
\text { SMAD3, SMAD4, mTOR, c-FOS, c-JUN, PKA, } \\
\text { PKC, ERK, MyDD8, IRF1, G proteins, etc. }\end{array}$ \\
\hline microRNAs & & $\begin{array}{l}\text { miR-21, miR-181, Dicer (ribonuclease) identified } \\
\text { thus far }\end{array}$ \\
\hline
\end{tabular}

Nomenclature for growth factors, cytokines, chemokines, receptors, and transcription factors are per The Human Gene Compendium, GeneCards (see www.genecards.org).

DAMPs, danger-associated molecular patterns; PAMPs, pathogen-associated molecular patterns.

tracellular second messenger signaling cascades (Table 1), as reviewed elsewhere (Pekny and Nilsson 2005; Sofroniew 2009; Sofroniew and Vinters 2010; Kang and Hebert 2011). There is also emerging evidence that microRNAs (miR), such as miR-21 and miR-181 (Bhalala et al.2012; Hutchison et al. 2013), and miR regulatory enzymes, such as Dicer ( Tao et al. 2011), can modulate astrogliosis and reactive astrocyte functions, adding yet another level of potential regulation and specification of functions (Table 1). Thus, intrinsic properties of individual astrocytes, such as epigenetic mechanisms or genetic polymorphisms of receptors and second messengers and other regulators, may impact astrogliosis.
Selective Regulation of Specific Functions and Effects of Astrogliosis

There is increasing interest in dissecting molecular signaling mechanisms that regulate specific functions or effects of astrogliosis so that these might be targeted by specific therapeutic interventions (Sofroniew 2009; Hamby and Sofroniew 2010). Considerable information is now available, which reveals that some aspects of astrogliosis can be regulated by many pathways, whereas other aspects are regulated more selectively. For example, expression of structural filaments, such as GFAP or vimentin can be induced by signaling pathways associated with cAMP, STAT3, NF-кB, Rho-kinase, JNK, calci- 
um, and others (Pekny and Nilsson 2005; Sofroniew 2009; Middeldorp and Hol 2011; Gao et al. 2013). Similarly, astrocyte proliferation can be regulated by different extracellular signals including EGF, FGF, endothelin 1, Sonic Hedgehog, the serum proteins thrombin and albumin, and others, and by intracellular signals, such as Olig2, JNK pathway, and others (Levison et al. 2000; Gadea et al. 2008; Sofroniew 2009; Sirko et al. 2013). Other aspects of astrogliosis are regulated more selectively. For example, certain pro- and anti-inflammatory functions of astrocytes are regulated separately. Deletion or disruption of NF- $\mathrm{\kappa B}$ or SOC3 signaling pathways selectively in astrocytes diminishes recruitment of inflammatory cells after traumatic injury and autoimmune disease (Brambilla et al. 2005, 2009; Okada et al. 2006). Deletion of estrogen receptor- $\alpha$, but not estrogen receptor- $\beta$, selectively from astrocytes diminishes the antiinflammatory and neuroprotective effects of estrogen on autoimmune inflammation (Spence et al. 2011, 2013). In contrast, deletion of STAT3 or its associated membrane receptor GP130, markedly increases the spread of inflammation after traumatic injury, autoimmune disease, or infection (Okada et al. 2006; Drogemuller et al. 2008; Herrmann et al. 2008; Haroon et al. 2011; Wanner et al. 2013). Deletion of YLK-40/BRP39 , an astrocyte produced anti-inflammatory protein, exacerbates autoimmune disease in mice (Bonneh-Barkay et al. 2012). miR-181 decreases astrocyte production of inflammatory cytokines (TNF- $\alpha$, IL-1 $\beta$ ) and increases production of anti-inflammatory cytokines (IL10) (Hutchison et al. 2013). Thus, different signaling mechanisms regulate different aspects of pro- or anti-inflammatory functions of reactive astrocytes. From a different perspective, expression and function of the glutamate transporter GLT-1 can be modulated up or down during astrogliosis depending on the nature of the insult and time after the insult, and this regulation can impact outcome by influencing extracellular glutamate levels and the potential for neuronal excitotoxicity (Bianchi et al. 2013). The mTORAkt-NF- $\kappa \mathrm{B}$ pathway is one molecular signaling mechanism that can modulate astrocyte expression of Glt-1 (Pawlak et al. 2005; Faideau et al.
2010; Ji et al. 2013). Findings such as these support a model in which many different specific signaling mechanisms can trigger different specific molecular, morphological, and functional changes in reactive astrocytes. This model also predicts that the impact of astrogliosis on its host neural tissue and associated neural functions will not be uniform or stereotypic, but will vary in a context-specific manner as determined by the specific signaling mechanisms associated with specific insults and the environment in which they are occurring.

\section{BENEFICIAL FUNCTIONS AND MALADAPTIVE EFFECTS OF ASTROGLIOSIS}

In response to the many signaling events just described, reactive astrocytes have the potential to release a large variety of molecules that impact nearby cells (Fig. 1B; Table 2). These molecules can exert many different functions and effects. For much of its greater than 100-year history, astrogliosis has been regarded by some

Table 2. Selected examples of effector molecular released by reactive astrocytes

\begin{tabular}{lc}
\hline Categories & \multicolumn{1}{c}{ Molecules } \\
\hline Cytokines & IL-6, IL-1 $\beta$, TNF- $\alpha$, INF- $\gamma$, \\
& CNTF, LIF, TGF- $\beta$, IL-11, \\
Chemokines & IL-15 \\
& CXCL1, CXCL9, CXCL10, \\
& CCL5, CCL7, CCL8 \\
Growth factors and & BDNF, NGF, GDNF, THSP1, \\
other proteins & GPC4, GPC6, FGF2, \\
& PDGFb, BMP1, VEGF-A, \\
& pentraxin 3 (PT3), \\
Extracellular matrix & $\alpha$-crystallin B, etc. \\
& MMP, SEMA4A, TIMP1, \\
& chondroitin sulfate \\
Intermediate & proteoglycans (CSPG) \\
filaments & GFAP, vimentin, nestin \\
Transmitters & Glutamate (Glut), kynurenic \\
Small molecules & acid (KYN), D-serine \\
& NO, prostaglandin E (PGE), \\
\hline
\end{tabular}

Nomenclature as per The Human Gene Compendium, GeneCards (see www.genecards.org). 
M.V. Sofroniew

investigators as a reaction that is fundamentally harmful to functional recovery. This notion is refuted by a large volume of in vivo experimental studies from many laboratories, which show that in response to most if not all CNS insults, normally occurring astrogliosis is initiated as an adaptive, beneficial process directed at wound repair and tissue protection. Nevertheless, experimental studies have also established the potential for potentially harmful effects of astrogliosis under specific circumstances. Thus, it is important to understand that astrogliosis, like inflammation, exerts essential beneficial functions but can also give rise to maladaptive effects, and that these are regulated by specific signaling mechanisms that occur in specific contexts.

Transgenic loss-of-function models have been particularly useful in identifying beneficial functions and harmful effects of astrogliosis and astrocyte scar formation (Sofroniew 2005; Sofroniew and Vinters 2010). For example, transgenic ablation or prevention of astrogliosis or astrocyte scar formation causes increased inflammation and tissue damage and worsens functional outcome in all CNS insult models studies thus far, including traumatic injury, ischemic injury (stroke), infection, autoimmune inflammation, and neurodegenerative disorders (Nawashiro et al. 1998; Bush et al. 1999; Faulkner et al. 2004; Myer et al. 2006; Drogemuller et al. 2008; Herrmann et al. 2008; Li et al. 2008; Voskuhl et al. 2009; Haroon et al. 2011; Macauley et al. 2011; Wanner et al. 2013). Nevertheless, transgenic studies also reveal the potential for certain aspects of astrogliosis to exacerbate inflammation after traumatic injury or autoimmune challenge (Brambilla et al. 2005, 2009; Spence et al. 2011, 2013). Other studies show that controlling astrocyte $\mathrm{pH}$ can reduce glutamate excitotoxicity during ischemia (Beppu et al. 2014; Sloan and Barres 2014). Large-scale gene expression evaluations show that inflammatory mediators can drive astrocyte transcriptome profiles toward proinflammatory and potentially cytotoxic phenotypes (Hamby et al. 2012; Zamanian et al. 2012) that may be beneficial in microbial infection but may be detrimental if triggered during sterile (uninfected) tissue responses to trauma, stroke, degenerative disease, or autoimmune attack, as discussed below (Sofroniew 2013).

Perhaps the most widely regarded potential detrimental effect of astrogliosis is the inhibition of axon regeneration by astrocyte scars after CNS damage (Silver and Miller 2004). Nevertheless, this potential detrimental effect must be viewed in the context of the essential protective roles that astrocyte scars perform in restricting inflammation and protecting healthy tissue adjacent to lesions as discussed above. Simply preventing or removing astrocyte scars is likely to do more harm than good. Attempts to manipulate scar formation with the intention of improving axon regeneration will likely need to be very specifically targeted and controlled, and, given the multiple and complex causes for failure of CNS axon regeneration (Brosius Lutz and Barres 2014), are unlikely to be effective on their own.

Together, such observations provide compelling evidence that astrogliosis exerts a variety of beneficial functions that are essential for limiting tissue damage and preserving neurological function after CNS insults but that astrogliosis also has the potential to exert detrimental effects as determined by specific signaling mechanisms. Recognition of these concepts sets the stage for dissecting the physiology and pathophysiology of astrogliosis to identify and ameliorate the consequences of astrocyte dysfunction.

\section{DYSFUNCTIONS OF ASTROCYTES AND ASTROGLIOSIS: TOWARD A CONCEPT OF ASTROCYTE PATHOPHYSIOLOGY AND ASTROCYTOPATHIES}

There is increasing evidence for a concept of astrocytopathies in which a disruption of normal astrocyte functions in healthy tissue is the primary cause of neurological dysfunction and disease (Verkhratsky et al. 2012). The argument can be made that a full concept of astrocytopathies will also include disorders caused by altering the process of astrogliosis (Fig. 3). As discussed above, a strictly negative connotation of astrogliosis is being replaced by a balanced view of astrogliosis as an adaptive process generated and conserved by evolution, which serves es- 


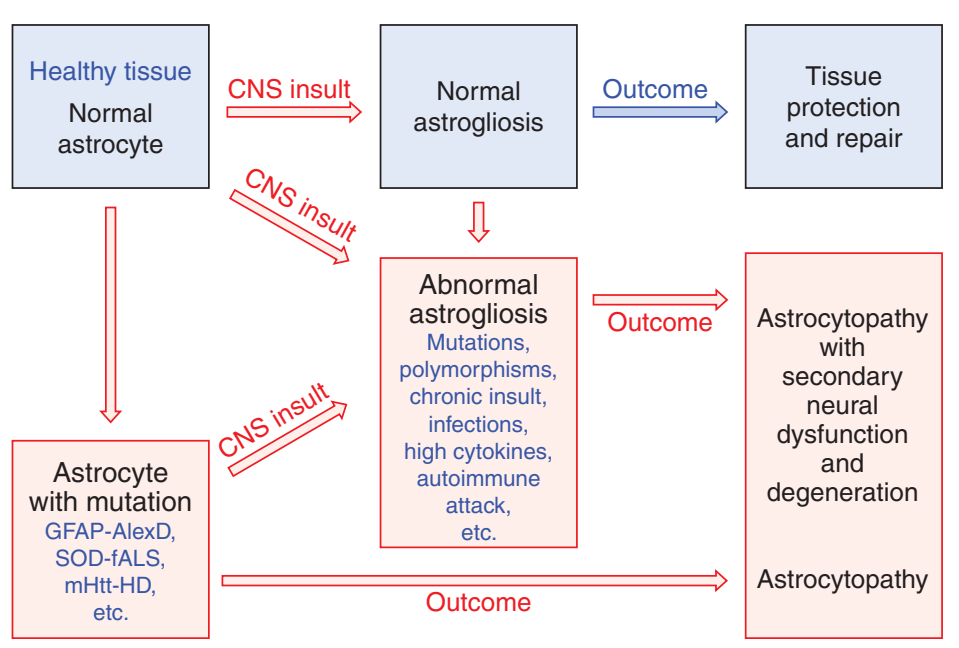

Figure 3. Schema that distinguishes normal from abnormal astrogliosis and depicts different pathways that can lead to astrocytopathies.

sential repair and tissue protective functions, but that in certain situations can show harmful effects as a direct consequence of contextinappropriate signaling interactions. This perspective allows a conceptual framework for astrogliosis that includes both adaptive (beneficial) and maladaptive (harmful) forms of astrogliosis, and allows for consideration of multiple ways in which dysfunctions of astrogliosis could be primary causes of, or major contributors to, CNS disorders. Recognizing and understanding the potential for dysfunction of astrogliosis (Fig. 3) is likely to be fundamental to dissecting the cellular mechanisms underlying a variety of CNS disorders. The next sections discuss clinical and experimental examples of ways in which primary dysfunctions of astrocytes or of astrogliosis could precipitate, or contribute to, neurological dysfunction and disease.

\section{ASTROCYTE GENE MUTATIONS}

There are now several examples of genetic mutations that cause astrocyte dysfunctions that can lead, or contribute significantly, to neuronal dysfunction and degeneration, which in turn lead to neurological symptoms and disease. One first example, Alexander disease, is caused by a dominant mutation of the GFAP gene, which is expressed in the CNS only by astroglia. Patients show macrocephaly, severe neurological dysfunction, seizures, psychomotor disturbances, and premature death (Brenner et al. 2001; Messing et al. 2012). A second well, studied example is a familial form of amyotrophic lateral sclerosis (ALS) caused by a dominant gain-of-function mutation of the gene-encoding superoxide dismutase (SOD) that leads to the production of molecules that are toxic to motor neurons. Targeting mutant SOD selectively to astrocytes in transgenic mice is sufficient to cause neuronal dysfunction and degeneration (Lobsiger and Cleveland 2007; Nagai et al. 2007; Yamanaka et al. 2008). Nevertheless, in the full disease, mutant SOD produced in neurons also contributes to neuronal degeneration. In addition to these examples, there is recent evidence from experimental animal models that the genetic mutation associated with Huntington's disease causes dysfunction of astrocytes in ways that may contribute significantly to neurological symptoms and neurodegeneration. In Huntington's disease, astrocytes as well as neurons accumulate nuclear inclusions of mutant huntingtin protein $(\mathrm{mHtt})$. In transgenic mouse models, astrocytes with $\mathrm{mHtt}$ down-regulate the potassium channel $\mathrm{K}_{\mathrm{ir}} 4.1$, leading to increased extracellular potassium and increased neuronal excitability, which is likely to contribute to and exacerbate direct effects of mHtt on neurons (Tong et al. 2014). It 
M.V. Sofroniew

is noteworthy that these effects occur at early stages of symptom onset and in the absence of astrocyte changes associated with astrogliosis (Tong et al. 2014). It is important to emphasize that in all of these conditions, the detrimental effects on neuronal function and neurotoxicity are caused by genetically mutated and abnormal astrocytes rather than by the normal process of astrogliosis. Thus, astrocyte dysfunction that is harmful can occur in the absence of astrogliosis, and astrogliosis can occur without causing harm (Fig. 3).

\section{ASTROCYTE MOLECULAR POLYMORPHISMS}

An intriguing and potentially important question is the degree to which genetic polymorphisms could alter functions of astrocytes and astrogliosis. Available evidence suggests that this is likely to be the case. Apolipoprotein E (APOE) polymorphisms influence the risk of Alzheimer's disease, with isoform APOE4 increasing risk and APOE2 reducing risk. Studies using various transgenic mouse models show that astrocyte-secreted APOE4, but not APOE3 or APOE2, is associated with increased blood-brain barrier leak and increased neuronal degeneration (Bell et al. 2012) in a manner that may be relevant to Alzheimer's disease pathology (Zlokovic 2011). The notion that astrocyte intrinsic molecular polymorphisms might impact astrocyte functions in disease-relevant ways is further supported by the example of genetic mutations or experimental gene manipulations that modulate astrocytes and astrogliosis in ways that alter neuronal function or cause neurodegeneration as discussed above.

\section{ASTROGLIOSIS AND CNS INFLAMMATION}

Inflammation is an important component of the multicellular response to CNS damage and disease that can markedly influence the balance between tissue loss and preservation (Burda and Sofroniew 2014). There is now both clinical and experimental evidence that astrocytes are critical regulators of CNS inflammation. From a clinical perspective, recent findings show that
CNS autoimmune disease that selectively damages astrocytes has a more severe course than autoimmune disease that involves other cellular targets. Neuromyelitis optica (NMO) is a CNS inflammatory demyelinating disease with pronounced tissue destruction and severe symptoms that can include vision loss and paralysis. NMO has been causally associated with autoantibodies to aquaporin-4 (AQP4) on astrocytes that mediate compliment-mediated astrocyte lysis (Lennon et al. 2005; Roemer et al. 2007). Both clinical course and tissue lesions are more severe in patients that have autoimmune-mediated CNS demyelination associated with antiAQP4 autoantibodies compared with patients with antimyelin autoantibodies (Kitley et al. 2014; Sato et al. 2014). These findings strongly suggest that AQP4 is not simply a passive autoimmune CNS antigen, but that disruption of astrocyte functions by AQP4 antibodies exacerbates the disease process and worsens the outcome. This notion is consistent with experimental evidence that astrocytes critically restrict the spread of inflammation during autoimmune CNS attack (Voskuhl et al. 2009; Haroon et al. 2011). From an experimental perspective, it is well documented that astrocytes can produce a wide variety of pro- or anti-inflammatory molecules and that specific molecular regulators modulate specific astrocyte functions in ways can increase or limit CNS inflammation. Astrocytes produce not only a wide range of chemokines that open the blood-brain barrier (Argaw et al. 2012) and attract inflammatory cells (Hamby et al. 2012; Zamanian et al. 2012; Sofroniew 2013) but also molecules that can exert potent suppressive effects on inflammatory cells (Kostianovsky et al. 2008). Astrocyte scars play critical roles in limiting the spread of inflammatory cells from damage areas into neighboring healthy tissue after trauma or autoimmune attack (Bush et al. 1999; Voskuhl et al. 2009; Wanner et al. 2013). Modulation of specific astrocyte functions can either attenuate or exacerbate CNS inflammation (Okada et al. 2006; Herrmann et al. 2008; Brambilla et al. 2009), opening the door for gain or loss of astrocyte functions to impact CNS inflammation (Dong and Benveniste 2001; Sofroniew 2009). Together, these 
findings provide compelling clinical and experimental evidence that dysfunction of astrogliosis can exacerbate CNS inflammation and the spread of tissue damage in various ways and contexts.

\section{ASTROCYTE-MEDIATED NON-CELL- AUTONOMOUS NEURONAL DYSFUNCTION OR DEGENERATION}

There are many ways that loss of astrocyte functions or disruption of astrogliosis could lead to non-cell-autonomous neuronal dysfunction or degeneration. In healthy neural tissue, astrocytes play critical roles for normal neuronal function including homeostasis of extracellular fluid, ions, and transmitters, regulation of blood flow, energy provision, and interactions with synapses as reviewed elsewhere (Barres 2008; Sofroniew and Vinters 2010). In damaged neural tissue, reactive astrocytes play critical roles in neuroprotection, blood-brain barrier repair, and regulation of inflammation (Sofroniew and Vinters 2010; Burda and Sofroniew 2014). Thus, perhaps not surprisingly, experimentally induced loss of specific functions of astrocytes or astrogliosis can cause neuronal dysfunction and degeneration and worsen outcome after CNS trauma, ischemia, or autoimmune attack. A pioneering example is that selective deletion of the astrocyte glutamate uptake transporter, Glt-1 (EAAT2) will lead to seizures and excitotoxic neuronal death (Rothstein et al. 1996). Further examples include (1) astrocyteselective expression of mutant SOD causes neuronal dysfunction and degeneration (Lobsiger and Cleveland 2007; Nagai et al. 2007; Yamanaka et al. 2008), (2) astrocyte-selective deletion of the endoribonuclease, Dicer, cases of severe ataxia, progressive cerebellar degeneration, seizures, and premature death (Tao et al. 2011), and (3) astrocyte-selective deletion of the Wnt signaling molecule adenopolyposis coli causes delayed degeneration of cerebellar Purkinje neurons (Wang et al. 2011). Experimental disruption of various astrocyte signaling molecules can alter astrogliosis and cause neuronal dysfunction and tissue degeneration during traumatic injury, ischemia, andautoimmuneattack (Okada et al. 2006; Drogemuller et al. 2008; Herrmann et al. 2008; Li et al. 2008; Haroon et al. 2011). It is also intriguing to consider whether chronic astrogliosis with long-term overexpression of GFAP may be sufficient to cause cellular dysfunction and degeneration. This possibility is raised not only by the clinical example Alexander disease with mutated GFAP discussed above, but also by evidence that chronic high level expression of normal GFAP may cause pathology (Messing et al. 1998). Chronic astrogliosis can occur in neurodegenerative disorders or when CNS insults are comorbid with infections as discussed below, and its effects warrant further study. Nonetheless, it deserves emphasis that, after acute insults, such as single traumatic or ischemic events that are not comorbid with infections, astrogliosis in perilesion tissue resolves relatively rapidly (within weeks) and GFAP levels return to normal or low levels. Last, an exciting new area pioneered by the Barres laboratory is astrocyte induction and pruning of neuronal synapses during development and in adult neural plasticity (Clarke and Barres 2013). Mice deficient in astrocyte-specific phagocytic pathways fail to develop normal connections and retain excess synapses (Chung et al. 2013). The impact of normal or anomalous astrogliosis on astrocyte interactions with synapses remains to be determined but has the potential to open new ways of thinking about certain CNS disorders.

\section{PERIPHERAL INFECTIONS AND MOLECULAR SIGNALS THAT SKEW ASTROGLIOSIS TOWARD CYTOTOXICITY}

Peripheral infections can generate high levels circulating cytokines and inflammatory mediators, such as microbial PAMPs, which can gain access to CNS lesions with compromised blood-brain barrier (Burda and Sofroniew 2014). Exposure of astrocytes to PAMPs, such as lipopolysaccharide and certain cytokines, drives astrocyte transcriptome profiles toward a proinflammatory and cytotoxic potential (Hamby et al. 2012; Zamanian et al. 2012; Sofroniew 2013). This response is not surprising because limiting infection spread is likely to have shaped the evolution of injury responses 
M.V. Sofroniew

in all tissues including the CNS (Burda and Sofroniew 2014). If sterile CNS insults are comorbid with peripheral infections, there is a strong possibility that the molecular expression profile and function of reactive glia may be altered in ways that can exacerbate tissue damage and compromise neural repair (Burda and Sofroniew 2014). In keeping with these experimental findings, clinical epidemiological observations indicate that peripheral infections have a negative impact on neurological outcome after spinal cord injury (Failli et al. 2012). The potential impact of concurrent low-grade infections, or even of microbiome (Heintz and Mair 2014), on astrogliosis and responses to CNS insults is likely to be an interesting area of future research.

\section{ASTROGLIOSIS AND NEURONAL FUNCTION AND BEHAVIOR}

Astrogliosis has the potential to modify astrocyte functions in ways that influence neuronal functions, including behaviors. In the healthy CNS, astrocytes (1) maintain the extracellular homeostasis of $\mathrm{K}^{+}$, water, $\mathrm{pH}$, and transmitter uptake (Halassa and Haydon 2010; Jin et al. 2013), (2) may modulate synaptic activity directly via release of "gliotransmitters" (Volterra and Meldolesi 2005; Halassa and Haydon 2010; Hamilton and Attwell 2010; Henneberger et al. 2010), and (3) take part in the formation and pruning of synapses (Chung et al. 2013; Clarke and Barres 2013). Functional changes undergone by reactive astrocytes in response to specific molecular triggers can impact neurons via (1) down-regulation of glutamine synthase associated with reduced inhibitory synaptic currents in local neurons (Ortinski et al. 2010), (2) increased expression of xCT (Slc7a11), a cysteine-glutamate transporter associated with increased glutamate signaling, seizures, and excitotoxicity (Jackman et al. 2010; Buckingham et al. 2011), and (3) changes in the expression of multiple GPCRs and G proteins and calcium signaling evoked by their ligands (Hamby et al. 2012). There is increasing evidence for astrocyte participation in complex behaviors including sleep (Halassa and Haydon 2010), pain (Hansen and Malcangio 2013), mood, depression (Ha- lassa and Haydon 2010; Paradise et al. 2012; Czeh and Di Benedetto 2013; Martin et al. 2013), and certain childhood behavioral disorders with altered synapse development (Stephan et al. 2012; Clarke and Barres 2013). Astrocytes are primary targets of cytokines and inflammatory mediators increasingly implicated not only in sickness behaviors, such as social withdrawal, reduced activity, loss of appetite, and cognitive disturbances, but also in certain kinds of sleep disturbances, mood disturbances, major depressive disorders, and CNS developmental disorders (Dantzer et al. 2008; Dowlati et al. 2010; Irwin and Cole 2011; Jurgens et al. 2012; Patterson 2012; Sofroniew 2013). Modulation of astrocyte physiology and function by cytokines and inflammatory mediators has the potential to impact such behaviors (Miller and O'Callaghan 2005; Sofroniew 2013). Although such interactions are only beginning to be studied, early information suggests a strong potential for identifying new ways in which CNS cellular responses to injury or disease impact systems-level neural functions and behaviors (Sofroniew 2013).

\section{ASTROGLIOSIS AS A THERAPEUTIC TARGET}

There is increasing recognition that molecular mechanisms associated with specific functions and effects of astrocytes and astrogliosis may be potential targets for novel therapeutic strategies for CNS disorders (Hamby and Sofroniew 2010). As emphasized throughout this article, astrogliosis exerts essential beneficial functions but can also give rise to harmful effects in specific contexts as determined by specific signaling events. Thus, useful therapeutic strategies will need to be specifically targeted so as to preserve or augment beneficial effects of astrogliosis while blocking or reducing harmful ones. The once prevalent view that wholesale blockade of astrogliosis or astroglial scar formation could be a therapeutic strategy is no longer tenable and would likely do more harm than good. Specific aspects of astrogliosis that are being explored as potential targets for therapeutic manipulations include mechanisms that regulate glutamate, enzymes that generate or neutralize reactive ox- 
ygen species, and production of certain cytokines (Hamby and Sofroniew 2010). Although detailed examination is beyond the scope of this article, astrocyte regulation of glutamate clearance and the potential role of astrocytes and astrogliosis in glutamate excitotoxicity after ischemia (Beppu et al. 2014; Sloan and Barres 2014) and during neurodegenerative disease (Maragakis and Rothstein 2006) are of particular interest. Pharmacological manipulation of expression or activity of astrocyte glutamate transporters is being explored, and molecules have been identified that enhance astrocyte-mediated glutamate uptake sufficiently to provide neuroprotection in animal models of stroke and neurodegenerative disorders (Rothstein et al. 2005; Fontana et al. 2007).

\section{CONCLUDING REMARKS}

Astrogliosis in response to CNS damage and disease has been recognized for well over 100 years. Recent progress shows that astrogliosis is a complex and multifaceted process that can range from subtle and reversible alterations in gene expression and morphology to the pronounced and long-lasting changes associated with scar formation. Responses of astrocytes to CNS insults are controlled in a context-dependent manner by specific signaling mechanisms that mediate numerous essential beneficial functions, but under certain circumstances can lead to harmful effects. Simplistic notions that astrogliosis and scar formation are maladaptive, uniformly detrimental responses, and that complete blockade of reactive astrogliosis per se will be beneficial, are refuted by findings from many laboratories. Essential functions of astrogliosis and scar formation include the protection of neural cells, the restriction of inflammation and infection, and the preservation of tissue and function. Dysfunctions of astrocytes and astrogliosis can also occur, either through gain of abnormal effects or loss of normal functions, and contribute to, or be primary causes of, a variety of CNS disorders. As a result, astrocytes and astrogliosis are increasingly recognized as potential targets for novel therapeutic strategies. Effective therapies will need to be directed at augmenting or attenuating specific aspects of astrogliosis.

\section{ACKNOWLEDGMENTS}

Work in the author's laboratory is supported by grants from the National Institutes of Health, The Dr. Miriam and Sheldon G. Adelson Medical Foundation, and Wings for Life.

\section{REFERENCES}

Aldrich A, Kielian T. 2011. Central nervous system fibrosis is associated with fibrocyte-like infiltrates. Am J Pathol 179: 2952-2962.

Anderson MA, Ao Y, Sofroniew MV. 2014. Heterogeneity of reactive astrocytes. Neurosci Lett 565C: 23-29.

Argaw AT, Asp L, Zhang J, Navrazhina K, Pham T, Mariani JN, Mahase S, Dutta DJ, Seto J, Kramer EG, et al. 2012. Astrocyte-derived VEGF-A drives blood-brain barrier disruption in CNS inflammatory disease. J Clin Invest 122: $2454-2468$.

Bardehle S, Kruger M, Buggenthin F, Schwausch J, Ninkovic J, Clevers H, Snippert HJ, Theis FJ, Meyer-Luehmann M, Bechmann I, et al. 2013. Live imaging of astrocyte responses to acute injury reveals selective juxtavascular proliferation. Nat Neurosci 16: 580-586.

Barnabe-Heider F, Goritz C, Sabelstrom H, Takebayashi H, Pfrieger FW, Meletis K, Frisen J. 2010. Origin of new glial cells in intact and injured adult spinal cord. Cell Stem Cell 7: 470-482.

Barres BA. 2008. The mystery and magic of glia: A perspective on their roles in health and disease. Neuron 60: 430440.

Bell RD, Winkler EA, Singh I, Sagare AP, Deane R, Wu Z, Holtzman DM, Betsholtz C, Armulik A, Sallstrom J, et al. 2012. Apolipoprotein E controls cerebrovascular integrity via cyclophilin A. Nature 485: 512-516.

Beppu K, Sasaki T, Tanaka KF, Yamanaka A, Fukazawa Y, Shigemoto R, Matsui K. 2014. Optogenetic countering of glial acidosis suppresses glial glutamate release and ischemic brain damage. Neuron 81: 314-320.

Bhalala OG, Pan L, Sahni V, McGuire TL, Gruner K, Tourtellotte WG, Kessler JA. 2012. microRNA-21 regulates astrocytic response following spinal cord injury. J Neurosci 32: 17935-17947.

Bianchi MG, Bardelli D, Chiu M, Bussolati O. 2013. Changes in the expression of the glutamate transporter EAAT3/ EAAC1 in health and disease. Cell Mol Life Sci 71: 20012015.

Bonneh-Barkay D, Wang G, Laframboise WA, Wiley CA, Bissel SJ. 2012. Exacerbation of experimental autoimmune encephalomyelitis in the absence of breast regression protein 39/chitinase 3-like 1. J Neuropathol Exp Neurol 71: 948-958.

Brambilla R, Bracchi-Ricard V, Hu WH, Frydel B, Bramwell A, Karmally S, Green EJ, Bethea JR. 2005. Inhibition of astroglial nuclear factor- $\mathrm{\kappa}$ reduces inflammation and 
M.V. Sofroniew

improves functional recovery after spinal cord injury. $J$ Exp Med 202: 145-156.

Brambilla R, Persaud T, Hu X, Karmally S, Shestopalov VI, Dvoriantchikova G, Ivanov D, Nathanson L, Barnum SR, Bethea JR. 2009. Transgenic inhibition of astroglial NF-кB improves functional outcome in experimental autoimmune encephalomyelitis by suppressing chronic central nervous system inflammation. J Immunol 182: 2628-2640.

Brenner M, Johnson AB, Boespflug-Tanguy O, Rodriguez D Goldman JE, Messing A. 2001. Mutations in GFAP, encoding glial fibrillary acidic protein, are associated with Alexander disease. Nat Genet 27: 117-120.

Brosius Lutz A, Barres BA. 2014. Contrasting the glial response to axon injury in the central and peripheral nervous systems. Dev Cell 28: 7-17.

Buckingham SC, Campbell SL, Haas BR, Montana V, Robel S, Ogunrinu T, Sontheimer H. 2011. Glutamate release by primary brain tumors induces epileptic activity. Nat Med 17: $1269-1274$.

Buffo A, Rite I, Tripathi P, Lepier A, Colak D, Horn AP, Mori T, Gotz M. 2008. Origin and progeny of reactive gliosis: A source of multipotent cells in the injured brain. Proc Natl Acad Sci 105: 3581-3586.

Bundesen LQ, Scheel TA, Bregman BS, Kromer LF. 2003. Ephrin-B2 and EphB2 regulation of astrocyte-meningeal fibroblast interactions in response to spinal cord lesions in adult rats. J Neurosci 23: 7789-7800.

Burda JE, Sofroniew MV. 2014. Reactive gliosis and the multicellular response to CNS damage and disease. Neuron 81: 229-248.

Bush TGNP, Horner CH, Polito A, Ostenfeld T, Svendsen CN, Mucke L, Johnson MH, Sofroniew MV. 1999. Leukocyte infiltration, neuronal degeneration and neurite outgrowth after ablation of scar-forming, reactive astrocytes in adult transgenic mice. Neuron 23: 297-308.

Chung WS, Clarke LE, Wang GX, Stafford BK, Sher A, Chakraborty C, Joung J, Foo LC, Thompson A, Chen C, et al. 2013. Astrocytes mediate synapse elimination through MEGF10 and MERTK pathways. Nature 504: 394-400.

Clarke LE, Barres BA. 2013. Emerging roles of astrocytes in neural circuit development. Nat Rev Neurosci 14: 311321.

Czeh B, Di Benedetto B. 2013. Antidepressants act directly on astrocytes: Evidences and functional consequences. Eur Neuropsychopharmacol 23: 171-185.

Dantzer R, O'Connor JC, Freund GG, Johnson RW, Kelley KW. 2008. From inflammation to sickness and depression: When the immune system subjugates the brain. Nat Rev Neurosci 9: 46-56.

Dong Y, Benveniste EN. 2001. Immune function of astrocytes. Glia 36: 180-190.

Dowlati Y, Herrmann N, Swardfager W, Liu H, Sham L, Reim EK, Lanctot KL. 2010. A meta-analysis of cytokines in major depression. Biol Psychiatry 67: 446-457.

Drogemuller K, Helmuth U, Brunn A, Sakowicz-Burkiewicz M, Gutmann DH, Mueller W, Deckert M, Schluter D. 2008. Astrocyte gp130 expression is critical for the control of Toxoplasma encephalitis. J Immunol 181: 26832693.
Faideau M, Kim J, Cormier K, Gilmore R, Welch M, Auregan G, Dufour N, Guillermier M, Brouillet E, Hantraye P, et al. 2010. In vivo expression of polyglutamine-expanded huntingtin by mouse striatal astrocytes impairs glutamate transport: A correlation with Huntington's disease subjects. Hum Mol Genet 19: 3053-3067.

Failli V, Kopp MA, Gericke C, Martus P, Klingbeil S, Brommer B, Laginha I, Chen Y, DeVivo MJ, Dirnagl U, et al. 2012. Functional neurological recovery after spinal cord injury is impaired in patients with infections. Brain 135: $3238-3250$.

Faulkner JR, Herrmann JE, Woo MJ, Tansey KE, Doan NB, Sofroniew MV. 2004. Reactive astrocytes protect tissue and preserve function after spinal cord injury. J Neurosci 24: $2143-2155$.

Fontana AC, de Oliveira Beleboni R, Wojewodzic MW, Ferreira Dos Santos W, Coutinho-Netto J, Grutle NJ, Watts SD, Danbolt NC, Amara SG. 2007. Enhancing glutamate transport: mechanism of action of Parawixinl, a neuroprotective compound from Parawixia bistriata spider venom. Mol Pharmacol 72: 1228-1237.

Gadea A, Schinelli S, Gallo V. 2008. Endothelin-1 regulates astrocyte proliferation and reactive gliosis via a JNK/ c-Jun signaling pathway. J Neurosci 28: 2394-2408.

Gao K, Wang CR, Jiang F, Wong AY, Su N, Jiang JH, Chai RC, Vatcher G, Teng J, Chen J, et al. 2013. Traumatic scratch injury in astrocytes triggers calcium influx to activate the JNK/c-Jun/AP-1 pathway and switch on GFAP expression. Glia 61: 2063-2077.

Garcia AD, Petrova R, Eng L, Joyner AL. 2010. Sonic Hedgehog regulates discrete populations of astrocytes in the adult mouse forebrain. J Neurosci 30: 13597-13608.

Halassa MM, Haydon PG. 2010. Integrated brain circuits: Astrocytic networks modulate neuronal activity and behavior. Annu Rev Physiol 72: 335-355.

Hamby ME, Sofroniew MV. 2010. Reactive astrocytes as therapeutic targets for CNS disorders. Neurotherapeutics 7: 494-506.

Hamby ME, Coppola G, Ao Y, Geschwind DH, Khakh BS, Sofroniew MV. 2012. Inflammatory mediators alter the astrocyte transcriptome and calcium signaling elicited by multiple G-protein-coupled receptors. J Neurosci 32: 14489-14510.

Hamilton NB, Attwell D. 2010. Do astrocytes really exocytose neurotransmitters? Nat Rev Neurosci 11: 227-238.

Hansen RR, Malcangio M. 2013. Astrocytes-multitaskers in chronic pain. Eur J Pharmacol 716: 120-128.

Haroon F, Drogemuller K, Handel U, Brunn A, Reinhold D, Nishanth G, Mueller W, Trautwein C, Ernst M, Deckert M, et al. 2011. Gp130-dependent astrocytic survival is critical for the control of autoimmune central nervous system inflammation. J Immunol 186: 6521-6531.

Heintz C, Mair W. 2014. You are what you host: Microbiome modulation of the aging process. Cell 156: 408-411.

Henneberger C, Papouin T, Oliet SH, Rusakov DA. 2010. Long-term potentiation depends on release of D-serine from astrocytes. Nature 463: 232-236.

Herrmann JE, Imura T, Song B, Qi J, Ao Y, Nguyen TK, Korsak RA, Takeda K, Akira S, Sofroniew MV. 2008 STAT3 is a critical regulator of astrogliosis and scar for- 
mation after spinal cord injury. J Neurosci 28: 72317243.

Hutchison ER, Kawamoto EM, Taub DD, Lal A, Abdelmohsen K, Zhang Y, Wood WH 3rd, Lehrmann E, Camandola S, Becker KG, et al. 2013. Evidence for miR-181 involvement in neuroinflammatory responses of astrocytes. Glia 61: 1018-1028.

Irwin MR, Cole SW. 2011. Reciprocal regulation of the neural and innate immune systems. Nat Rev Immunol 11: 625-632.

Jackman NA, Uliasz TF, Hewett JA, Hewett SJ. 2010. Regulation of system $\mathrm{x}_{\mathrm{c}}{ }^{-}$activity and expression in astrocytes by interleukin-1ß: Implications for hypoxic neuronal injury. Glia 58: 1806-1815.

Ji YF, Zhou L, Xie YJ, Xu SM, Zhu J, Teng P, Shao CY, Wang Y, Luo JH, Shen Y. 2013. Upregulation of glutamate transporter GLT- 1 by mTOR-Akt-NF- $\kappa$ B cascade in astrocytic oxygen-glucose deprivation. Glia 61: 1959-1975.

Jin BJ, Zhang H, Binder DK, Verkman AS. 2013. Aquaporin4-dependent $\mathrm{K}^{+}$and water transport modeled in brain extracellular space following neuroexcitation. J Gen Physiol 141: 119-132.

Jurgens HA, Amancherla K, Johnson RW. 2012. Influenza infection induces neuroinflammation, alters hippocampal neuron morphology, and impairs cognition in adult mice. J Neurosci 32: 3958-3968.

Kang W, Hebert JM. 2011. Signaling pathways in reactive astrocytes, a genetic perspective. Mol Neurobiol 43: 147154.

Kitley J, Waters P, Woodhall M, Leite MI, Murchison A, George J, Kuker W, Chandratre S, Vincent A, Palace J. 2014. Neuromyelitis optica spectrum disorders with aquaporin-4 and myelin-oligodendrocyte glycoprotein antibodies: A comparative study. JAMA Neurol 71: 273-283.

Kostianovsky AM, Maier LM, Anderson RC, Bruce JN, Anderson DE. 2008. Astrocytic regulation of human monocytic/microglial activation. J Immunol 181: 5425-5432.

Lennon VA, Kryzer TJ, Pittock SJ, Verkman AS, Hinson SR. 2005. IgG marker of optic-spinal multiple sclerosis binds to the aquaporin-4 water channel. J Exp Med 202: 473477.

Levison SW, Jiang FJ, Stoltzfus OK, Ducceschi MH. 2000. IL-6-type cytokines enhance epidermal growth factorstimulated astrocyte proliferation. Glia 32: 328-337.

Li L, Lundkvist A, Andersson D, Wilhelmsson U, Nagai N, Pardo AC, Nodin C, Stahlberg A, Aprico K, Larsson K, et al. 2008. Protective role of reactive astrocytes in brain ischemia. J Cereb Blood Flow Metab 28: 468-481.

Lobsiger CS, Cleveland DW. 2007. Glial cells as intrinsic components of non-cell-autonomous neurodegenerative disease. Nat Neurosci 10: 1355-1360.

Macauley SL, Pekny M, Sands MS. 2011. The role of attenuated astrocyte activation in infantile neuronal ceroid lipofuscinosis. J Neurosci 31: 15575-15585.

Magnus T, Carmen J, Deleon J, Xue H, Pardo AC, Lepore AC, Mattson MP, Rao MS, Maragakis NJ. 2008. Adult glial precursor proliferation in mutant $\mathrm{SOD} 1^{\mathrm{G} 93 \mathrm{~A}}$ mice. Glia 56: $200-208$.
Maragakis NJ, Rothstein JD. 2006. Mechanisms of Disease: Astrocytes in neurodegenerative disease. Nat Clin Pract Neurol 2: 679-689.

Martin JL, Magistretti PJ, Allaman I. 2013. Regulation of neurotrophic factors and energy metabolism by antidepressants in astrocytes. Curr Drug Targets 14: 1308-1321.

Meletis K, Barnabe-Heider F, Carlen M, Evergren E, Tomilin N, Shupliakov O, Frisen J. 2008. Spinal cord injury reveals multilineage differentiation of ependymal cells. PLoS Biol 6: e182.

Messing A, Head MW, Galles K, Galbreath EJ, Goldman JE, Brenner M. 1998. Fatal encephalopathy with astrocyte inclusions in GFAP transgenic mice. Am J Pathol 152: 391-398.

Messing A, Brenner M, Feany MB, Nedergaard M, Goldman JE. 2012. Alexander disease. J Neurosci 32: 5017-5023.

Middeldorp J, Hol EM. 2011. GFAP in health and disease. Prog Neurobiol 93: 421-443.

Miller DB, O'Callaghan JP. 2005. Depression, cytokines, and glial function. Metabolism 54: 33-38.

Myer DJ, Gurkoff GG, Lee SM, Hovda DA, Sofroniew MV. 2006. Essential protective roles of reactive astrocytes in traumatic brain injury. Brain 129: 2761-2772.

Nagai M, Re DB, Nagata T, Chalazonitis A, Jessell TM, Wichterle H, Przedborski S. 2007. Astrocytes expressing ALSlinked mutated SOD1 release factors selectively toxic to motor neurons. Nat Neurosci 10: 615-622.

Nawashiro H, Messing A, Azzam N, Brenner M. 1998. Mice lacking GFAP are hypersensitive to traumatic cerebrospinal injury. Neuroreport 9: 1691-1696.

Okada S, Nakamura M, Katoh H, Miyao T, Shimazaki T, Ishii K, Yamane J, Yoshimura A, Iwamoto Y, Toyama Y, et al. 2006. Conditional ablation of Stat3 or Socs3 discloses a dual role for reactive astrocytes after spinal cord injury. Nature Med 12: 829-834.

Ortinski PI, Dong J, Mungenast A, Yue C, Takano H, Watson DJ, Haydon PG, Coulter DA. 2010. Selective induction of astrocytic gliosis generates deficits in neuronal inhibition. Nat Neurosci 13: 584-591.

Paradise MB, Naismith SL, Norrie LM, Graeber MB, Hickie IB. 2012. The role of glia in late-life depression. Int Psychogeriatr 24: 1878-1890.

Patterson PH. 2012. Maternal infection and autism. Brain Behav Immun 26: 393.

Pawlak J, Brito V, Kuppers E, Beyer C. 2005. Regulation of glutamate transporter GLAST and GLT-1 expression in astrocytes by estrogen. Brain Res Mol Brain Res 138: 1-7.

Pekny M, Nilsson M. 2005. Astrocyte activation and reactive gliosis. Glia 50: 427-434.

Reilkoff RA, Bucala R, Herzog EL. 2011. Fibrocytes: Emerging effector cells in chronic inflammation. Nat Rev Immunol 11: 427-435.

Roemer SF, Parisi JE, Lennon VA, Benarroch EE, Lassmann H, Bruck W, Mandler RN, Weinshenker BG, Pittock SJ Wingerchuk DM, et al. 2007. Pattern-specific loss of aquaporin-4 immunoreactivity distinguishes neuromyelitis optica from multiple sclerosis. Brain 130: $1194-$ 1205.

Rothstein JD, Dykes-Hoberg M, Pardo CA, Bristol LA, Jin L, Kuncl RW, Kanai Y, Hediger MA, Wang Y, Schielke JP, et al. 1996. Knockout of glutamate transporters reveals a 
M.V. Sofroniew

major role for astroglial transport in excitotoxicity and clearance of glutamate. Neuron 16: 675-686.

Rothstein JD, Patel S, Regan MR, Haenggeli C, Huang YH, Bergles DE, Jin L, Dykes Hoberg M, Vidensky S, Chung DS, et al. 2005. $\beta$-Lactam antibiotics offer neuroprotection by increasing glutamate transporter expression. $\mathrm{Na}$ ture 433: 73-77.

Sato DK, Callegaro D, Lana-Peixoto MA, Waters PJ, de Haidar Jorge FM, Takahashi T, Nakashima I, Apostolos-Pereira SL, Talim N, Simm RF, et al. 2014. Distinction between MOG antibody-positive and AQP4 antibodypositive NMO spectrum disorders. Neurology 82: 474481.

Silver J, Miller JH. 2004. Regeneration beyond the glial scar. Nat Rev Neurosci 5: 146-156.

Sirko S, Behrendt G, Johansson PA, Tripathi P, Costa M, Bek S, Heinrich C, Tiedt S, Colak D, Dichgans M, et al. 2013. Reactive glia in the injured brain acquire stem cell properties in response to Sonic Hedgehog. Cell Stem Cell 12: $426-439$.

Sloan SA, Barres BA. 2014. The detrimental role of glial acidification during ischemia. Neuron 81: 221-223.

Sofroniew MV. 2005. Reactive astrocytes in neural repair and protection. Neuroscientist 5: 400-407.

Sofroniew MV. 2009. Molecular dissection of reactive astrogliosis and glial scar formation. Trends Neurosci 32: 638 647.

Sofroniew MV. 2013. Multiple roles for astrocytes as effectors of cytokines and inflammatory mediators. Neuroscientist 20: 160-172.

Sofroniew MV, Vinters HV. 2010. Astrocytes: Biology and pathology. Acta Neuropathol 119: 7-35.

Spence RD, Hamby ME, Umeda E, Itoh N, Du S, Wisdom AJ, Cao Y, Bondar G, Lam J, Ao Y, et al. 2011. Neuroprotection mediated through estrogen receptor- $\alpha$ in astrocytes. Proc Natl Acad Sci 108: 8867-8872.

Spence RD, Wisdom AJ, Cao Y, Hill HM, Mongerson CR, Stapornkul B, Itoh N, Sofroniew MV, Voskuhl RR. 2013. Estrogen mediates neuroprotection and anti-inflammatory effects during EAE through $\mathrm{ER} \alpha$ signaling on astrocytes but not through ER $\beta$ signaling on astrocytes or neurons. J Neurosci 33: 10924-10933.

Stephan AH, Barres BA, Stevens B. 2012. The complement system: An unexpected role in synaptic pruning during development and disease. Annu Rev Neurosci 35: 369389.

Tao J, Wu H, Lin Q, Wei W, Lu XH, Cantle JP, Ao Y, Olsen RW, Yang XW, Mody I, et al. 2011. Deletion of astroglial dicer causes non-cell-autonomous neuronal dysfunction and degeneration. J Neurosci 31: 8306-8319.

Tong X, Ao Y, Faas GC, Nwaobi SE, Xu J, Haustein MD, Anderson MA, Mody I, Olsen ML, Sofroniew MV, et al.
2014. Astrocyte Kir4.1 ion channel deficits contribute to neuronal dysfunction in Huntington's disease model mice. Nat Neurosci 17: 694-703.

Tsai HH, Li H, Fuentealba LC, Molofsky AV, Taveira-Marques R, Zhuang H, Tenney A, Murnen AT, Fancy SP, Merkle F, et al. 2012. Regional astrocyte allocation regulates CNS synaptogenesis and repair. Science 337: 358-362.

Verkhratsky A, Sofroniew MV, Messing A, deLanerolle NC, Rempe D, Rodriguez JJ, Nedergaard M. 2012. Neurological diseases as primary gliopathies: A reassessment of neurocentrism. ASN Neuro 4.

Volterra A, Meldolesi J. 2005. Astrocytes, from brain glue to communication elements: The revolution continues. Nat Rev Neurosci 6: 626-640.

Voskuhl RR, Peterson RS, Song B, Ao Y, Morales LB, TiwariWoodruff S, Sofroniew MV. 2009. Reactive astrocytes form scar-like perivascular barriers to leukocytes during adaptive immune inflammation of the CNS. J Neurosci 29: 11511-11522.

Wang X, Imura T, Sofroniew MV, Fushiki S. 2011. Loss of adenomatous polyposis coli in Bergmann glia disrupts their unique architecture and leads to cell nonautonomous neurodegeneration of cerebellar Purkinje neurons. Glia 59: 857-868.

Wanner IB, Anderson MA, Song B, Levine J, Fernandez A, Gray-Thompson Z, Ao Y, Sofroniew MV. 2013. Glial scar borders are formed by newly proliferated, elongated astrocytes that interact to corral inflammatory and fibrotic cells via STAT3-dependent mechanisms after spinal cord injury. J Neurosci 33: 12870-12886.

White RE, McTigue DM, Jakeman LB. 2010. Regional heterogeneity in astrocyte responses following contusive spinal cord injury in mice. J Comp Neurol 518: 1370-1390.

Wilhelmsson U, Bushong EA, Price DL, Smarr BL, Phung V, Terada M, Ellisman MH, Pekny M. 2006. Redefining the concept of reactive astrocytes as cells that remain within their unique domains upon reaction to injury. Proc Natl Acad Sci 103: 17513-17518.

Yamanaka K, Chun SJ, Boillee S, Fujimori-Tonou N, Yamashita H, Gutmann DH, Takahashi R, Misawa H, Cleveland DW. 2008. Astrocytes as determinants of disease progression in inherited amyotrophic lateral sclerosis. Nat Neurosci 11: 251-253.

Zamanian JL, Xu L, Foo LC, Nouri N, Zhou L, Giffard RG, Barres BA. 2012. Genomic analysis of reactive astrogliosis. J Neurosci 32: 6391-6410.

Zhang Y, Barres BA. 2010. Astrocyte heterogeneity: An underappreciated topic in neurobiology. Curr Opin Neurobiol 20: 588-594.

Zlokovic BV. 2011. Neurovascular pathways to neurodegeneration in Alzheimer's disease and other disorders. Nat Rev Neurosci 12: 723-738. 


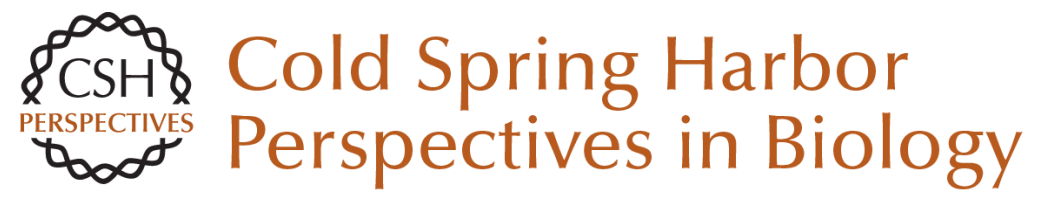

\section{Astrogliosis}

Michael V. Sofroniew

Cold Spring Harb Perspect Biol 2015; doi: 10.1101/cshperspect.a020420 originally published online November 7, 2014

\section{Subject Collection Glia}

The Nodes of Ranvier: Molecular Assembly and Maintenance

Matthew N. Rasband and Elior Peles

Microglia in Health and Disease

Richard M. Ransohoff and Joseph El Khoury

The Astrocyte: Powerhouse and Recycling Center Bruno Weber and L. Felipe Barros

Microglia Function in Central Nervous System

Development and Plasticity

Dorothy P. Schafer and Beth Stevens

Transcriptional and Epigenetic Regulation of Oligodendrocyte Development and Myelination in the Central Nervous System

Ben Emery and Q. Richard Lu

Origin of Microglia: Current Concepts and Past

Controversies

Florent Ginhoux and Marco Prinz

Glia Disease and Repair--Remyelination

Robin J.M. Franklin and Steven A. Goldman

\section{Astrocytes in Neurodegenerative Disease}

Hemali Phatnani and Tom Maniatis
Oligodendrocyte Development and Plasticity Dwight E. Bergles and William D. Richardson

Oligodendrocytes: Myelination and Axonal

Support Mikael Simons and Klaus-Armin Nave

Drosophila Central Nervous System Glia Marc R. Freeman

Perisynaptic Schwann Cells at the Neuromuscular

Synapse: Adaptable, Multitasking Glial Cells Chien-Ping Ko and Richard Robitaille

Astrocytes Control Synapse Formation, Function, and Elimination Won-Suk Chung, Nicola J. Allen and Cagla Eroglu

Schwann Cell Myelination James L. Salzer

Schwann Cells: Development and Role in Nerve Repair

Kristján R. Jessen, Rhona Mirsky and Alison C. Lloyd

Perineurial Glia

Sarah Kucenas

For additional articles in this collection, see http://cshperspectives.cshlp.org/cgi/collection/

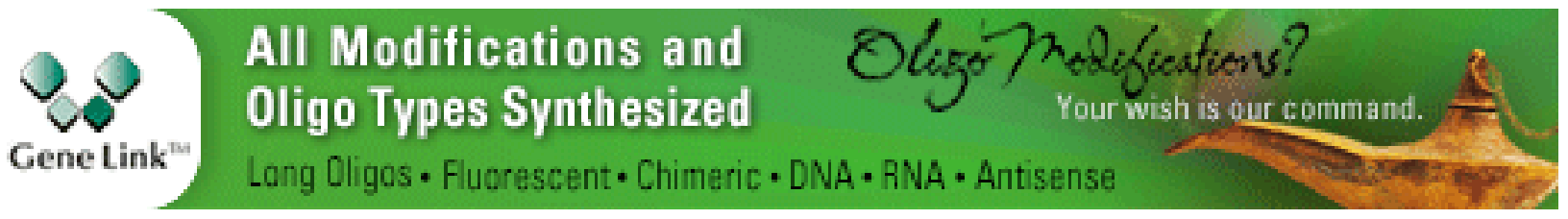

Copyright @ 2015 Cold Spring Harbor Laboratory Press; all rights reserved 